\title{
Papers
}

\section{Qualitative study of evidence based leaflets in maternity care}

Helen Stapleton, Mavis Kirkham, Gwenan Thomas

\begin{abstract}
Objective To examine the use of evidence based leaflets on informed choice in maternity services. Design Non-participant observation of 886 antenatal consultations. 383 in depth interviews with women using maternity services and health professionals providing antenatal care.

Setting Women's homes; antenatal and ultrasound clinics in 13 maternity units in Wales.

Participants Childbearing women and health professionals who provide antenatal care.

Intervention Provision of 10 pairs of Informed Choice leaflets for service users and staff and a training session in their use.

Main outcome measures Participants' views and commonly observed responses during consultations and interviews.

Results Health professionals were positive about the leaflets and their potential to assist women in making informed choices, but competing demands within the clinical environment undermined their effective use. Time pressures limited discussion, and choice was often not available in practice. A widespread belief that technological intervention would be viewed positively in the event of litigation reinforced notions of "right" and "wrong" choices rather than "informed" choices. Hierarchical power structures resulted in obstetricians defining the norms of clinical practice and hence which choices were possible. Women's trust in health professionals ensured their compliance with professionally defined choices, and only rarely were they observed asking questions or making alternative requests. Midwives rarely discussed the contents of the leaflets or distinguished them from other literature related to pregnancy. The visibility and potential of the leaflets as evidence based decision aids was thus greatly reduced.
\end{abstract}

Conclusions The way in which the leaflets were disseminated affected promotion of informed choice in maternity care. The culture into which the leaflets were introduced supported existing normative patterns of care and this ensured informed compliance rather than informed choice.

\section{Introduction}

The organisation and provision of maternity care in the United Kingdom was challenged when the Chang- ing Childbirth report recommended that it become more "woman centred." The 10 research based leaflets (Informed Choice) ${ }^{2}$ were developed by the Midwives Information and Resource Service to support consumer choice. ${ }^{3}$ The effectiveness of these leaflets has been studied in a randomised controlled trial which is reported separately. ${ }^{2}$ To understand the social context in which the leaflets were used we undertook qualitative research alongside, but independently of, the randomised trial.

Attitudes of staff are thought to influence the choices available to childbearing women $^{45}$ and decision making in clinical practice. ${ }^{6-8}$ Organisational culture affects the quality of health care..$^{9-11}$ "Socially complex interventions," ${ }^{12}$ such as the Informed Choice leaflets, should be evaluated within the context in which they are used and through a prudent combination of qualitative and quantitative methods. ${ }^{13} 14$

\section{Methods}

In the randomised controlled trial, 13 maternity units formed 10 clusters, five of which received the intervention of the Informed Choice leaflets between May and December $1998 .{ }^{3}{ }^{15}$ Four female midwifery researchers, including two of the authors (HS and GT), undertook non-participant observation and in depth interviews with health professionals and women, in both intervention and control maternity units (table). All the researchers kept detailed field notes for analysis. We used a grounded theory approach to data collection and analysis $^{16}$ and the software package QSR NUD*IST ${ }^{17}$ to organise and interrogate the datasets.

The combination of qualitative methods enabled us to examine the same issue from a range of different perspectives and to explore beyond "official" accounts

Summary of qualitative methods in study of informed choice in maternity services

\begin{tabular}{lcc} 
Respondents & $\begin{array}{c}\text { Episodes of } \\
\text { observation }\end{array}$ & \multicolumn{1}{c}{ Interviews } \\
\hline Childbearing women & 886 & 163 (85 antenatal, 78 postnatal) \\
\hline Midwives & 653 & 177 \\
\hline Obstetricians & 167 & 28 \\
\hline Obstetric ultrasonographers & 66 & 12 \\
\hline Obstetric anaesthetists & $\mathrm{NA}^{*}$ & 3 \\
\hline Total & 886 & 383 (17 conducted in Welsh) \\
\hline
\end{tabular}

*NA=not applicable as observation of women in labour was not undertaken.

\author{
Women's Informed \\ Childbearing and \\ Health Research \\ Group, School of \\ Nursing and \\ Midwifery, \\ University of \\ Sheffield, Sheffield \\ S3 7ND \\ Helen Stapleton \\ research midwife \\ Mavis Kirkham \\ professor of midwifery \\ Carmarthenshire \\ NHS Trust, West \\ Wales General \\ Hospital, \\ Carmarthen \\ SA31 2AF \\ Gwenan Thomas \\ midwife \\ Correspondence to: \\ H Stapleton \\ h.stapleton@ \\ sheffield.ac.uk
}


of choice and decision making from health professionals and childbearing women.

We obtained approval for the study from the local ethics committee.

\section{Observations}

We used observations of antenatal consultations (table) to identify how the leaflets were used and how informed choice and decision making occurred in practice. We made detailed field notes concerning setting, actions, words, and non-verbal cues of all present.

\section{Interviews}

We undertook face to face interviews using a semistructured format. We developed interview guides that were specific to the different participant groups, but all participants were invited to discuss the availability and quality of information, including the Informed Choice leaflets, receiving and conveying information, the meaning of informed choice, and the role of childbearing women in decision making. We also discussed inferences made by the researchers about behaviours and interactions during consultations. More than half of the interviews followed on from observation sessions, and this enabled us to explore issues, especially those of a sensitive nature, within the context of a previously established relationship.

\section{Sampling}

Our initial observation sample was "opportunistic," being determined by the staff on duty and whether

\section{Box 1: General views}

"... the best thing I've seen in terms of patient information ... it's the way we should all be going in the health services ... everyone, including health professionals, needs access to information of that quality" (obstetrician, intervention site)

"There are some ladies you don't want to be giving the leaflets to because you don't want them thinking they can have choices that aren't available. . There are some women who can't read for example ... and the young girls don't tend to be that interested" (community midwife, intervention site)

"They're good. They were really good. Especially the one about the positions. No one told me I could walk around last time. I didn't know you could stand up in labour. I thought it had to be lying on the bed. It were a real eye opener that one were ..." (17 year old unemployed woman, in the third trimester of pregnancy and expecting her second baby, when asked her opinion about the leaflets; field notes, intervention site)

"Here's a leaflet on ... [leaflet topic]. Have a read of it ... see what you think, it might give you some ideas... If you want to discuss anything, give us a ring" (observation of midwife interaction, intervention site)

"If I hadn't actually stopped to read them [Informed Choice leaflets] I'd never have appreciated how important they were ... The leaflets were just given to me ... When I started reading them I thought 'Oh this is what I've been looking for ...' I always had them with me when I went for my visits but nobody ever mentioned them, besides to say 'Have you got your leaflets?' But nobody ever discussed them" (service user, intervention site)

"The fact that they were called Informed Choice ... that's a very good title for them. They really push you to make an informed choice $\ldots$ The one about epidural really made me think. I didn't have one [epidural] this time ... I know I might not have needed one because it was my second [baby] but I didn't want one either because of what it said in the leaflet about it" (service user, intervention site)

"They were OK ... they were nothing 'wow' or anything ... they weren't anything new ... they didn't change my mind ... I already knew what I wanted to do" (service user, intervention site) they and their clients were willing to accommodate the researchers. We identified commonly observed responses, such as pregnant women expressing satisfaction with their care and complying with the choices offered to them, staff expressing concern about time pressures, and midwives describing the leaflets as useful tools. As the research progressed, we sampled more selectively to ensure that all women of childbearing age, all social classes, and various current and previous obstetric experiences were represented, together with women from minority groups. We sampled many more midwives than other health professionals because they provided most antenatal care and disseminated most of the leaflets. In an effort to observe and understand good practice we sampled a small number of midwives in all maternity units who were described by their managers as excellent in facilitating informed choice.

Towards the end of the intervention period we selected interviewees to confirm or refute emerging theory. As most staff and women tended to "go with the flow" of routine clinical practices, such sampling thus included women who questioned or declined the choices offered to them and staff who offered choices (and leaflets) withheld by colleagues. For midwives this revealed a link between practice and work setting. Midwives in community or domicilary settings were generally more knowledgeable about women's individual needs and seemed more willing to advocate on their behalf. They also tended to make more openings for women to voice their concerns. Hence, we identified interplay between hierarchy, power, and trust and the impact on information sharing between women and different groups of midwives. We continued to explore themes with all relevant participant groups until repeated, rather than new, information was forthcoming and theoretical saturation was achieved.

We removed identifying information from selected transcripts and shared them with members of the research team and outside experts. This guided future data collection, guarded against any researcher dominating the analytical process, and helped to ensure validity and reliability. ${ }^{18} 19$

\section{Results}

\section{The "invisible" leaflets}

Most health professionals initially expressed positive views about the principles underpinning the Informed Choice leaflets (box 1). Within practice settings, however, they were seldom used to maximum effect. Pragmatic usage resulted in many leaflets being withheld from women because staff disagreed with the contents of the leaflet or were concerned because some leaflets promoted choices that were unavailable locally. Some midwives also made assumptions about the ability and willingness of women to participate in decision making. These assumptions were sometimes incorrect.

The potential of the leaflets was further diluted because they were often given out "wrapped" within advertising materials or concealed within the maternity folder. During interviews, questions about the leaflets usually failed to elicit any response from most women. They often confused them with other information related to pregnancy or indeed denied having received them. It was often only after coaxing by 


\section{Box 2: Time pressures}

“... at the end of the day we are governed by time, we haven't hours and hours to spend with each person... It's been good having the [Informed Choice] leaflets ... it's a quick way out of it ... You can give them a leaflet and tell them to have a read of it" (midwife, intervention site)

"It's strange, but I found that there were often things that I felt I needed to know. I was never sure whether I should ask the midwife or not... sometimes they are busy aren't they? ... But if I did ask then they were brilliant... The information was there but you had to ask for it, you couldn't expect it to come pouring out" (service user, intervention site)

"I didn't feel happy throughout the pregnancy with the information I received ... I constantly felt they [midwives] did not have any time for me. I was given plenty of leaflets but not enough discussion. I was never in the consulting room for any longer than five minutes at any of my antenatal appointments" (service user, intervention site)

the researcher or after her suggestion that women look within their maternity folder that leaflets were discovered and some comments were forthcoming.

Midwives generally distributed leaflets in routine ways and were rarely observed differentiating them from other information they offered women. Health professionals were seldom observed discussing the leaflets with women or asking them if they understood the information or found it useful. Women rarely initiated discussion about leaflet topics. A few women were complimentary about the leaflets and thought that they had influenced their intentions. Most women, however, did not find them helpful in decision making.

\section{Health professionals under pressure}

Most health professionals reported feeling pressured by time constraints (box 2). Midwives in particular were concerned about assuming the role of "information broker" without preparation or the allocation of additional time. Midwives on intervention sites sometimes viewed the leaflets as a pragmatic solution to time pressures. Women were often observed accommodating health professionals by limiting their questions, but some expressed dissatisfaction when written information was used as an alternative to discussion.

\section{Lack of choice in practice}

Health professionals noted that many leaflets suggested choices that were not available at a local level (box 3). Furthermore, some technological interventions, such as ultrasound scanning and monitoring in labour, have become so routine in maternity care that health professionals no longer perceive them as optional. Women sometimes made choices on the basis of their previous experiences of childbirth but were often met with resistance if their preferences contradicted established clinical norms. Staff sometimes expressed a strong dislike for an option covered by the leaflets to the extent that distribution of some leaflets was terminated on some sites. Women tended to comply with the suggestions of health professionals, and, unless openings were made, they rarely instigated discussion about their own preferences.

\section{Technology and litigation}

Researchers observed health professionals driving decision making towards technological intervention by conveying information which either minimised the risk of the intervention or emphasised the potential for harm without the intervention. This seemed to make it difficult for women to hear alternative messages, even from obstetricians. Fear of litigation promoted notions of "right" choices with which clinicians felt clinically secure and which they thought would afford them protection against litigation. Midwives occasionally expressed frustration when such imperatives, rather than evidence based information or client choice, determined the options available. Some women were aware of the influence of technological imperatives on the attitudes of health professionals, and they occasionally experienced this as bullying. Some views are shown in box 4 .

\section{Hierarchy, power, and trust}

We observed a strong hierarchy within the maternity services, with obstetricians at the top, midwives and health professionals other than doctors in the middle,

\section{Box 3: Choices}

"When you go for your hospital appointment they'll do a little scan just to see how far on you are... They have the portable scanners in all the rooms so it's very quick. Then at 18 weeks you'll have your big scan ... the detailed anomaly scan" (observation of midwife interaction, intervention site)

Researcher: "Was monitoring in labour ever discussed with you?" Woman: "No ... not really... They gave me that leaflet [Informed Choice leaflet $\left.2^{2}\right]$ and told me to read it but they never said anything about what would happen in labour. They said they had to do a little trace when I first came in and then I think they just forgot to take it off" (service user, intervention site)

"With my first, I was monitored the whole time. I didn't realise that you could move round. Nobody explained that to me, but the second time around I knew that you didn't have to do it. I think the second time round I knew you had an option. You're stronger. You're a stronger person. You know what to expect" (service user, control site)

"When I declined the dating scan, the receptionist said: 'Oh, I'll just go and see if you're allowed.' ... That did annoy me, you're not allowed this, you're not allowed that ... I didn't have a dating scan and I had a hassle over it right up to the end" (service user, control site)

"Home deliveries are for pizzas and nothing else ... women who choose to have home deliveries are very irresponsible ... I know you can't stop them but I don't agree with them. If something does go wrong you haven't got a hope" (registrar, intervention site)

Researcher: "Was the option of having this baby at home discussed?" Woman: "No ... it did cross my mind. I thought I wouldn't mind considering a home birth but it wasn't mentioned. It was either a choice of [hospital X or hospital Y]."

Researcher: "And were you given this leaflet?" [researcher shows woman the Informed Choice leaflet on place of birth]

Woman: "Oh yes. I had that one but she [midwife] never discussed it with me ... I thought she would ask me at the next visit if I'd read it but she never did so I just dropped it really. It wasn't that important ... I'm quite happy with [hospital X]. I don't know why she bothered giving it me. I did wonder that..." (interview with women expecting her third baby; intervention site) 


\section{Box 4: Technology}

"Giving them a choice is not enough. They need to know the reality behind it [vaginal breech delivery] ... about the head getting stuck definitely. You can give them scare stories but you don't even have to do that. You just have to mention a complication. Something like the baby might die..." (registrar, intervention site)

The following excerpt, taken from field notes made during a routine antenatal consultation, describes an interaction between a female registrar and a working class woman with a breech presentation in her first pregnancy. The woman and her mother have both made it clear that they consider an elective caesarean section to be the only safe delivery option. The registrar attempts to present an alternative perspective:

"Well ... women still die from caesarean sections ... It's a big operation and not without its risks and complications ... What I want you to do, every morning, lunch time and tea time, is get on the floor on all fours for 10 minutes, with your forearms on the floor and your bum in the air. Do that for 10 minutes three times a day. It might encourage that baby to turn round."

The woman laughs; her mother looks disgusted (field notes, control site)

"You see there is a need for the legal document and I think rightly so because it's useful to have this in practice where there is a lot of litigation... A patient comes in, you think the foetus is OK because the Pinard has recorded everything as normal. Then the next moment you get sudden deceleration... You have no excuse for not having a tracing. If a tracing had been done perhaps it would have shown ... [an] increased risk in utero..." (consultant obstetrician, intervention site)

“... they will always guide a woman towards elective section for breech even if she wants to try for a vaginal delivery ... They're so geared towards an elective section ... Even when the woman comes in with an undiagnosed breech in labour, fully dilated, ready to push, they'll do an emergency section at that point rather than let her deliver vaginally. So what's the point of giving a leaflet [number $\left.9^{2}\right]$... What's the point of giving them information about choices they haven't got?" (midwife, intervention site)

"He [the obstetrician] is a real Jekyll and Hyde that one. He was fine as long as he thought I was going to the hospital but as soon as I said I wanted to have the baby at home it was all about haemorrhage and the risks to the baby. If anything went wrong it would be my own fault; it would be on my head. It was horrible. I came home and cried" (service user, intervention site)

"I do think you can be bullied into things, particularly if you're not strong minded about what you want. It's quite easy for them to bully you, they say things like 'The baby will die' if you don't do so and so. Or: 'You'll be in danger.' It's very easy for them because you don't really understand the medical stuff" (service user, intervention site)

\section{Discussion}

This qualitative study of evidence based leaflets for pregnant women found that they did not promote informed choice. This was related to time pressures on staff working within a culture that supported existing normative patterns of care rather than informed choice. The hierarchical power structures within the maternity services, and the framing of information in favour of particular options, ensured compliance with the "right" choice.

Health professionals' initial views of the Informed Choice leaflets were generally positive but the ways in which leaflets were distributed or withheld, however, severely diluted their potential benefits. Health professionals, pressured by time and concerned about litigation, rarely discussed the content of the leaflets or promoted their difference from other literature. The resulting invisibility helps to explain why only $70 \%$ of women in the intervention sites reported receiving a minimum of one leaflet. ${ }^{15}$ Such findings are not unique to maternity care or to the use of evidence based decision aids. ${ }^{21}$

The way in which information is presented influences decision making ${ }^{22}$ and competing "hierarchies of evidence" ${ }^{14}$ are known to reduce the credibility of some healthcare choices. Passive dissemination of information is ineffective in changing the behaviour of health professionals. ${ }^{23}$ Choices that are offered but not actively supported by staff are rarely taken up by pregnant women. ${ }^{24}$ The absence of opportunities for discussion ${ }^{25}$ that we observed is also likely to have hindered women in using the leaflets to make (informed) decisions.

The organisational and hierarchical structure of the maternity services worked against maximising the potential of the leaflets. The relative lack of continuity of care observed throughout the study made it difficult for women to follow up on issues raised in a previous consultation or to initiate discussion on leaflets and other topics related to pregnancy. Lack of continuity also precluded the formation of trusting relationships thought necessary to facilitate informed choice. ${ }^{26}$ Societal and medical expectations tend to normalise technological interventions, and some choices promoted in the leaflets, such as whether to have ultrasound scanning or electronic monitoring in labour, were rarely available in practice because the technology had long been integrated into routine care. Health professionals generally felt responsible for anything that went wrong in maternity care, and a widespread fear of litigation caused many to promote technological interventions, even when they were contradicted by the evidence base of the Informed Choice leaflets.

Choice and decision making seemed to be heavily circumscribed by the pressures and norms of the local obstetric culture. The researchers observed little diversity in clinical practice between individual practitioners or maternity units. Inequalities in power and status were observed to be potent forces in maintaining the status quo, and this made it difficult to promote (informed) choice. As reported elsewhere ${ }^{27} 28$ midwives were observed to "frame" information and "steer" women towards making the "right" decisions to "protect" themselves and their clients from the of such support often reported feeling undermined and were sometimes mistrusted by health professionals. 
consequences of inadvertently disrupting the status quo. Informed choice was therefore equated with making the locally defined "right" choice in accordance with the authoritative knowledge and experience of senior obstetricians. Unequal power relations resulted in bias towards the "objective" knowledge of health professionals and marginalised women's subjective knowledge. ${ }^{30}$ Hence, power differentials served to reinforce informed compliance with the right choice rather than encourage informed choice. Hierarchical structures in the maternity services also made it difficult for lower ranking practitioners to support women in going against these right choices. There was little evidence to suggest that concepts such as partnership ${ }^{20}$ or shared decision making ${ }^{31}$ were understood by staff who generally were observed to seek women's compliance with the professionally defined right choices.

Childbearing women generally complied with expected norms in their encounters with staff, who they perceived as busy people with many demands on their time. Our results show that cultural barriers within the maternity services encourage informed compliance, even though staff adopted the rhetoric of informed choice.

\section{Conclusions and implications}

The results of this study are not specific to maternity units in Wales as similar issues have been identified in units that independently purchased the leaflets. ${ }^{15}$ These leaflets are unlikely to promote informed choice in maternity care unless they are introduced as part of a coherent strategy addressing power imbalances and the ambiguities currently underpinning choice. The concept of informed choice carries great potential to resolve many of the issues faced by maternity services today, with informed choice and partnership in decision making lessening the burden of responsibility presently experienced by health professionals. From this and other research we can begin to understand the barriers facing the implementation of research based

\section{What is already known on this topic}

Informed Choice leaflets are widely used in maternity care but little is known about their ability to influence informed choice and decision making

High quality information is essential for promoting informed choice but is insufficient by itself

\section{What this study adds}

Time constraints and other pressures on health professionals resulted in a lack of discussion of the content of the leaflets

Fear of litigation, power hierarchies, and the technological imperative in maternity care limited the choices available

Health professionals promoted normative practices rather than choice, and as women valued their opinions this led to the promotion of informed compliance rather than informed choice

\section{Box 5: Knowing your place}

"In the later stage they [midwives] don't seem to have as much say in your care really. It's down to the consultant whether he chooses, or whether he recommends to you if the baby should be born [induced]" (service user, intervention site)

“... you wish your midwife was given more power than they are $\ldots$ it comes to a certain question which they have to refer to a doctor and you think, but they know! They're in there ... But they still have to defer. They should be empowered to do this" (service user, control site)

“... the Informed Choice leaflets actually put midwives between a rock and a very hard place... It's unreal to encourage women to go against local policies and guidelines when we all know that if she takes that line, she'll be given a really hard time, especially by the medical profession ... I mean we've still got women in this area being threatened and struck off GPs' lists just for saying they are considering a home birth, for God's sake ... and they will have to live with the consequences of making a choice ... for a lot longer than I will" (community midwife, intervention site)

"So I was still thinking should I or shouldn't I have an epidural? This leaflet [leaflet number $6^{2}$ ] was guiding me whether I should or shouldn't. In the end I had no choice anyway. When I went to deliver my babies, I had no choice. They were deciding how I was going to deliver my babies and told me I needed an epidural. I didn't know whether I did or not" (service user, intervention site)

“... it's a consultant led service. You may disagree with what your consultant says but if you're working for that consultant that's what you've got to tell the patient ... Whether you like it or not that's what you do, whether you totally disagree, you've got to do it" (registrar, intervention site)

"They [midwives] become your friends don't they? It's not just about the pregnancy. They start to know what your husband does, what you did, what you worked as; and it's the trust thing. Going back to that word again, they become part of your life and you do put your trust in that person" (service user, intervention site)

"You start to doubt yourself if you think differently to the midwife or whoever is advising you, and if you don't agree, you think, 'Oh! I must be wrong.' Or at least, I did" (service user, control site)

"We let them [women] do what they want to do and then when things go wrong we get sued. We are ... afraid to go against the women's wishes ... [But] you get very skilled at smelling a rat. We know now when trouble is approaching and that woman [who had requested something with which the obstetrician disagreed] smells like trouble" (obstetrician, intervention site)

evidence and the use of decision aids for informed choice in various clinical settings. ${ }^{14} 2126$ The additional barriers we have identified are unlikely to be unique to maternity care. Their removal, however, will entail considerable cultural change at all levels of the maternity services.

We thank the women, health professionals and managers, representatives of consumer groups and other organisations, and non-NHS antenatal teachers who contributed to the research. We also thank the project advisory group; colleagues and friends who assisted the research team in many ways including critical reading of work in progress; the National Childbirth Trust, who kindly agreed to withhold dissemination of a range of their leaflets on pregnancy related topics until our study was complete; and MIDIRS (Midwives Information and Resource Service) for answering our many queries and for withholding sales of the Informed Choice leaflets to control maternity units participating in the research until the end of the intervention period.

Contributors: HS advised on the study design, coordinated the qualitative research team, assisted in the qualitative data collection, conducted most of the data analysis, and participated in writing the paper. MK designed the study, supervised the qualitative data collection, and assisted in data analysis and writing the paper. GT contributed to the qualitative data collection and analysis. Donna Mead, Barbara Bale, Laurence Moseley, Sandy 
Kirkman, Heather Rothwell, David Cohen, and Penny Curtis were members of the research team for the wider study. Jane Durell was the project administrator. HS and MK are the guarantors.

Funding: Department of Health. The Welsh Office funded the translation into Welsh of the women's version of the Informed Choice leaflets and the transcribing of interviews conducted in Welsh.

Competing interests: None declared.

1 Department of Health. Changing childbirth. Report of the expert maternity group. London: HMSO, 1993.

2 O'Cathain A, Walters SJ, Nicholl JP, Thomas KJ, Kirkham M. Use of evidence based leaflets to promote informed choice in maternity care: randomised controlled trial in everyday practice. $B M J$ 2002;324:643-6.

3 Rosser J, Watt IS, Entwistle V. Informed choice initiative: an example of reaching users with evidence based information. I Clin Effect $1996 ; 1: 143-5$

4 Jones S, Sadler T, Low N, Blott M, Welch J. Does uptake of antenatal HIV testing depend on the individual midwife? Cross sectional study. BMJ 1998:316:272-3

5 McCrea BH, Wright ME, Murphy-Black T. Differences in midwives' approaches to pain relief in labour. Midwifery 1998;14:174-80.

6 Elwyn G, Edwards A, Gwyn R, Grol R. Towards a feasible model for shared decision making: focus group study with general practice registrars. $B M J$ 1999;319:753-6.

7 Charles C, Redko C, Whelan T, Gafni A, Reyno L. Doing nothing is not choice: lay constructions of treatment decision-making among women with early-stage breast cancer. Sociol Health Iln 1998;20:71-95.

8 Roter D. The medical visit context of treatment decision-making and the therapeutic relationship. Health Expect 2000;3:17-25.

9 Davies HT, Nutley SM, Mannion R. Organisational culture and quality of health care. Qual Health Care 2000;9:111-9.

10 Davies C. Gender and the professional predicament in nursing. Buckingham: Open University Press, 1995.

11 Murphy-Lawless J. Reading birth and death: a history of obstetric thinking. Cork: Cork University Press, 1998.

12 Wolff N. Randomised trials of socially complex interventions: promise or peril? J Health Serv Res Policy 2001;6:123-6.

13 Barbour RS. The case for combining qualitative and quantitative approaches in health services research. J Health Serv Res Policy 1999;4:3943.

14 Fitzgerald L, Ferlie E, Wood M, Hawkins C. Evidence into practice? An exploratory analysis of the interpretation of evidence In: Mark AL,
Dopson S, eds. Organisational behaviour in health care: the research agenda. London: Macmillan Business Press, 1999.

15 Kirkham M, Stapleton H, eds. Informed choice in maternity care: an evaluation of evidence based leaflets. York: NHS Centre for Reviews and Dissemination, 2001.

16 Glaser B, Strauss A. The discovery of grounded theory. New York: Aldine, 1967.

17 Gahan C, Hannibal M. Doing qualitative research using QSR NUD*IST. London: Sage, 1999.

18 Dingwall R. Accounts, interviews and observations. In: Miller G, Dingwal R, eds. Context and method in qualitative research. London: Sage, 1997.

19 Strauss A, Corbin J. Basics of qualitative research: grounded theory procedures and techniques. London: Sage, 1990.

20 Pairman S. Women-centred midwifery: partnerships or professional friendships? In: Kirkham M, ed. The midwife-mother relationship. London: Macmillan, 2000.

21 Holmes-Rovner M, Valade D, Orlowski C, Draus C, Nabozny-Valerio B, Keiser S. Implementing shared decision-making in routine practice: barriers and opportunities. Health Expect 2000;3:182-91.

22 Bekker H, Thornton JG, Airey CM, Connelly JB, Hewison J, Robinson $\mathrm{MB}$, et al. Informed decision making: an annotated bibliography and systematic review. Health Technol Assess 1999;3:1-156.

23 NHS Centre for Reviews and Dissemination, Royal Society of Medicine. Getting evidence into practice. Effect Health Care Bull 1999;5:1-16.

24 Machin D, Scamell A. Using ethnographic research to examine effects of 'informed choice'. Br J Midwifery 1998;6:304-9.

25 Proctor S. What determines quality in maternity care? Comparing the perceptions of childbearing women and midwives. Birth 1998;25:85-93.

26 Entwistle V, Sheldon TA, Sowden A, Watt IS. Evidence-informed patient choice. Practical issues of involving patients in decisions about health care technologies. Int J Technol Assess Health Care 1998;14:212-25.

27 Marteau T. Framing of information: its influence upon decisions of doctors and patients. Br J Soc Psychol 1989;28:89-94.

28 Press N, Browner CH. Why women say yes to prenatal diagnosis. Soc Sci Med 1997;45:979-89.

29 Levy V. Protective steering: a grounded theory study of the processes by which midwives facilitate informed choice in pregnancy. J Adv Nursing 1999;29:104-12.

30 Edwards NP. Women planning homebirths: their own views on their relationships with midwives. In: Kirkham M, ed. The midwife-mother relationship. London: Macmillan, 2000.

31 Charles C, Gafni A, Whelan T. Shared decision-making in the medical encounter: what does it mean? (or it takes at least two to tango). Soc Sci Med 1997:44:681-92

(Accepted 5 October 2001) 